\title{
ABSTRACTS FROM THE WORKSHOP ON HEARING IMPLANTS FOR OLDER ADULTS, NEW YORK CITY, 16-18 JANUARY 2014
}

\author{
Venue: Le Parker Meridien Hotel, New York City
}

Worldwide, the population is aging at an unprecedented rate and life expectancies are higher than ever before. Diseases of aging that have been associated with severe hearing impairment - from depression and dementia to increased risk of falls - can have devastating physical, emotional and financial consequences to individuals and society as a whole. The promise of restoring hearing and giving quality of life back to older adults with age-related hearing loss (ARHL) is a challenging and multi-faceted research topic. ARHL is currently one of the major handicaps found in elderly people, affecting more than $30 \%$ of the population over the age of 65 .

In January 2014, MED-EL GmbH organized an international workshop in New York City on Hearing Implants for Older Adults, which was attended by more than 120 outstanding international hearing professionals. Researchers from a range of hearing science disciplines presented data on the morphologic and physiologic bases of ARHL, geriatric audiology, cognition and hearing, the aging brain, electrophysiology, physical and perceptual changes of aging, hearing implant surgery, and outcomes as well as bal-

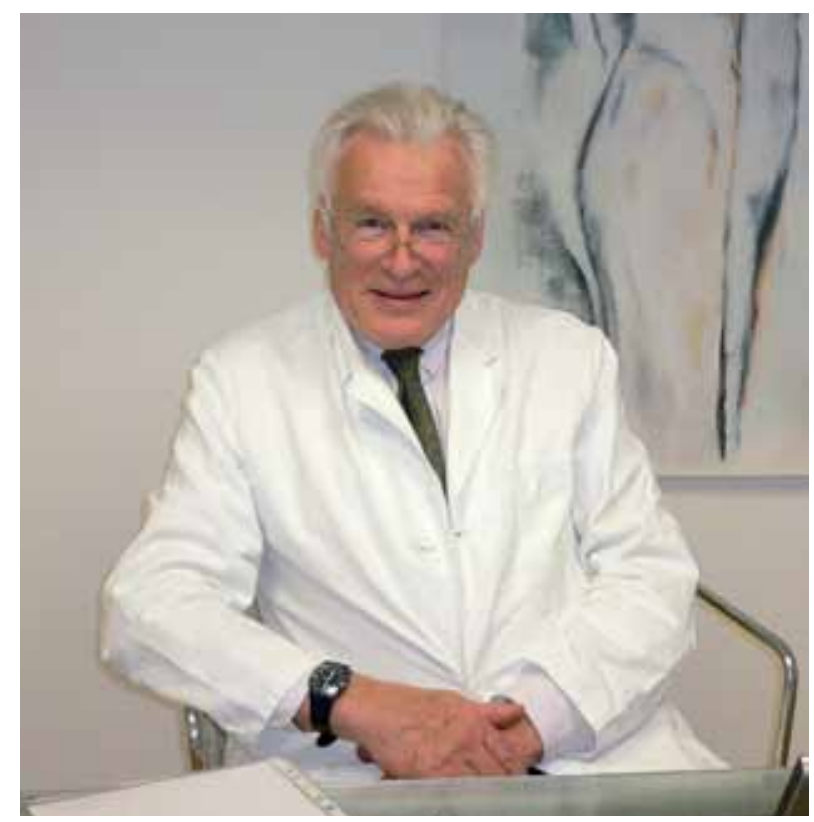
ance and fall risks.

An important topic discussed was hearing technologies as alternatives to help individuals suffering from severe ARHL who cannot achieve sufficient speech comprehension with conventional hearing aids. A combined electric-acoustic solution may be particularly suited for persons with ARHL because the hearing loss configuration typically presents with relatively good hearing in the low frequencies and poor hearing in the high frequencies, which qualifies candidates for electrical stimulation, either alone or in combination with acoustic amplification, while preserving low frequency hearing.

Further, recent clinical research has shown that there is no upper age limit to receiving hearing implants. Elderly cochlear implant users enjoy improved quality of life with similar performance outcomes to those achieved in younger adults, which is promising for people facing progressive and severe hearing loss.

ARHL is an exciting research topic that has been embraced by scientists from around the world. It is also an important public health issue that deserves attention from health care providers, policymakers and the media.

Christoph von Ilberg 


\section{Hearing Loss, Dementia and Public Health}

F. $\operatorname{Lin}^{1,2}$

\section{${ }^{1}$ Department of Otolaryngology-Head and Neck Surgery, The Johns Hopkins School of Medicine \\ ${ }^{2}$ Center on Aging and Health Johns Hopkins Medical Institutions}

Age-related hearing loss (ARHL) in older adults is often perceived as being an unfortunate but relatively inconsequential part of aging. However, the broader implications of hearing loss for the health and functioning of older adults are now beginning to surface in epidemiologic studies. I will discuss recent epidemiologic research demonstrating that hearing loss is independently associated with accelerated cognitive decline and an increased risk of incident dementia. I will then discuss more recent ongoing and planned studies investigating the role of hearing loss in accelerated volume loss in temporal brain regions. Results from analyses of several large epidemiologic datasets including the National Health and Nutritional Examination Surveys, the Health Aging and Body Composition Study, and the Baltimore Longitudinal Study of Aging will be presented.

\section{Development of a Cognitive Screening Test for the Severely Hearing Impaired}

\author{
V. Lin ${ }^{1,2}$, J. Chung 2 , D. Shipp ${ }^{1,2}$, M. Masellis ${ }^{3,4}$ \\ ${ }^{1}$ Cochlear Implant Program, Sunnybrook Health Sciences \\ Centre, Toronto, Ontario, Canada \\ ${ }^{2}$ Department of Otolaryngology-Head and Neck Surgery, \\ University of Toronto, Toronto, Ontario, Canada \\ ${ }^{3}$ Department of Neurology, University of Toronto, Toronto, \\ Ontario, Canada \\ ${ }^{4}$ Department of Neurology, Sunnybrook Health Sciences Centre, \\ Toronto, Ontario, Canada
}

Objective: With the aging of our population, we are increasingly encountering patients with mild cognitive impairment (MCI). However, all current and commonly utilized validated screening tools for cognitive testing rely on the ability for the patients to follow auditory verbal instructions. No clinical screening test for MCI is available for patients with severe hearing loss. Our objective is to validate a modified version of the Montreal Cognitive Assessment (MoCA) for use in patients with hearing impairment (MoCA-H).

Methods: Older-aged subjects were recruited from outpatient Otolaryngology clinics. Patients who volunteered for the study first underwent a battery of cognitive screening tools. Patients who passed the screen were then administered the MoCA-H. The MoCA-H was performed on 55 normal hearing subjects and 50 subjects with severe-toprofound hearing loss (HL). The regular MoCA was retested 6 months after MoCA-H was administered in a subset of normal hearing subjects.

Results: The average score of patients who underwent the MoCA-H was $26.6(\mathrm{SD}=2.04)$, consistent with the accepted normal cutoff of $>26 / 30$. No significant difference was demonstrated between the MoCA-H scores in those with and without HL. Similar scores were noted between the MoCA-H and MoCA in the subset of normal hearing subjects that were retested. Further subanalysis of results of the individual questions revealed small differences between the MoCA-H and MoCA.

Conclusions: The MoCA-H results of normal cognitively screen subjects have a high correlation to MoCA results. The MoCA-H is the only tool to detect MCI in patients with hearing impairment.

\section{Impact of Cochlear Implantation on Cognitive Factors in the Elderly}

I. Mosnier', JP. Bebear ${ }^{2}$, B. Fraysse B $^{3}$,

G. Lina-Granade ${ }^{4}$, M. Mondain ${ }^{5}$, P. Bordure ${ }^{6}$,

A. Robier ${ }^{7}$, B. Godey ${ }^{8}$, B. Meyer ${ }^{1}$, C. Poncet ${ }^{9}$,

D. Bouccara ${ }^{1}$, O. Sterkers ${ }^{1}$

${ }^{1}$ AP-HP, Hôpital Pitié-Salpétrière, Centre Référent des Implants Cochléaires et du Tronc Cérébral en Ile de France, Paris 75013,

France - UMR-S 867, Inserm / Université Paris 7 Denis

Diderot, Paris 75018, France (isabelle.mosnier@psl.aphp.fr)

${ }^{2}$ ORL, Hôpital Pellegrin, Bordeaux, France

${ }^{3}$ ORL, Hôpital Purpan, Toulouse, France

${ }^{4}$ ORL, Hôpital E. Hériot, Lyon, France

${ }^{5}$ ORL, Hôpital Gui de Chauliac, Montpellier, France

${ }^{6}$ ORL, Hôpital Hôtel Dieu, Nantes, France

${ }^{7}$ ORL, Hôpital Bretonneau, Tours, France

${ }^{8}$ ORL, Hôpital Pontchailloux, Rennes, France

${ }^{9}$ APHP, ORL, Hôpital Rotschild, Paris, France

Introduction: The prevalence of Alzheimer's disease and other dementia in the United States and Europa is estimated of approximately $5 \%$ for ages 70 to 79 , reaching about $40 \%$ over age 90 years. Epidemiologic studies estimated that the number of people with dementia would double every 20 years to 81 millions worldwide by 2040 .

Some prospective studies showed an independent association between peripheral hearing loss and dementia. Subjects with mild, moderate and severe hearing loss have a 2-, 3 - and 5-fold increased risk of developing dementia respectively, compared to subjects with normal hearing. Another prospective study reported that individuals having hearing loss demonstrate a $30 \%$ to $40 \%$ accelerated rate of cognitive decline and a $24 \%$ increased risk for incident cognitive impairment during a 6-year period compared to subjects with a normal hearing [Lin et al., Arch Neurol 2011]. In crosssectional studies including adults free of cognitive disabilities, the difference in cognitive performance associated with a $25 \mathrm{~dB}$ hearing loss is equivalent to the reduction associated with an age difference of 7 years. Despite these associations between hearing impairment and cognitive dysfunction, only eight studies published between 1990 and 2013 examine the impact of hearing rehabilitation on cognitive function, including only patients using hearing aids. Half of them show a beneficial effect of auditory rehabilitation on cognitive abilities. Others are unable to demonstrate an association between hearing aid use and cognitive measures. The impact of hearing rehabilitation using cochlear implants on cognitive function is not yet investigated. 
Objectives: To analyse the relationships between cognitive function and hearing rehabilitation using cochlear implants in patients over 65 years.

Study design: 95 patients were prospectively included. The mean age was 72 (range: 64-85). Patients were evaluated before implantation, 6 and 12 months after activation. Speech comprehension score was calculated using disyllabic words in quiet and at different SNR. Cognitive function was assessed using a battery of six tests evaluating attention, memory, orientation, executive function, mental flexibility and fluency. Quality of life was evaluated using NIJMEGEN questionnaire.

Results: Before cochlear implantation, 71 patients (76\%) had abnormal scores on at least one cognitive test and $43 \%$ of the population had 2 to 3 abnormal tests. Twelve months after CI, the number of patients with normal cognitive tests improved (39\%), the number of patients with one abnormal cognitive test remained stable and the number of patients with two and three abnormal tests decreased $\left(24 \%, \mathrm{P}<0.05, \chi^{2}\right)$. There was a significant improvement of the mean cognitive scores for all the tests in patients with initially abnormal results, as soon as 6 months after CI. Multivariate analysis revealed that only one cognitive test, the Letter fluency test, was a predictor of improvements in performance in noise $(\mathrm{P}=0.01$ at $\mathrm{SNR}+15 \mathrm{~dB}, \mathrm{P}=0.0007$ at $\mathrm{SNR}+10 \mathrm{~dB}, \mathrm{P}=0.019$ at $\mathrm{SNR}=5 \mathrm{~dB}$ ).

Conclusions: To conclude, hearing rehabilitation using cochlear implantation improved cognitive function in elderly patients as soon as 6 months after CI. In elderly patients, cognitive testing adapted to patients with hearing loss and exploring a broad range of cognitive function (such as the MOCA-test) is recommended before cochlear implantation. However, an effect of cognitive factors on post-operative speech performance was not observed in this study, except for the Letter fluency test. Further studies examining more precisely working memory and other specific factors for learning abilities have to be performed in order to evaluate if they can account for individual differences in speech performance after cochlear implantation, and help the speech therapy.

Keywords: Cochlear implant • elderly • cognitive function $\bullet$ predictive factors

\section{Hearing Health Care Challenges for Older Adults: Confronting and Overcoming the Barriers}

\section{B. Weinstein}

\section{City University of New York, USA}

Hearing loss is independently associated with a number of age related factors including functional and cognitive decline, increased rates of hospitalization, utilization of health care services, and a narrowing of social networks. Despite the fact that the need and desire for socialization assumes great import as we age especially in terms of mediating physical and psychologic well-being, the majority of older hearing impaired adults do not take advantage of available therapeutic treatments designed to promote social engagement. The fact that older adults, with a unique constellation of comorbidity, do not avail themselves of interventions that may enhance functioning, health related quality of life and prevent disability and functional decline, is a reality stakeholders must confront. Identifying and removing the multifactorial barriers to comprehensive hearing loss treatments is an important first step in promoting healthy aging. The obstacles which include competing risks, lack of awareness of the threats to health aging posed by hearing loss and the availability and value of available treatments, will be discussed as will approaches to translating this knowledge into practice.

\section{Hearing Loss Assessment in a Geriatric Setting}

\section{Lerch}

Helios Klinik, Schwerin, Germany

Background: In geriatric care nearly every third patient over the age of 70 and every second patient over the age of 75 is hearing impaired to such a degree that a reliable conversation cannot be achieved without the use of handicap-oriented communication strategies or amplification [Lerch/Decker-Maruska 2007]. Nevertheless in most hospital situations this handicap is not recognized, due to a lack of awareness on the part of the (nursing) staff and to the reluctance on the part of the patient to acknowledge his own deficiency due to the stigma the impaired hearing still carries. This situation leads to a dysfunctional communication, misunderstandings, aggression and non-compliance.

Especially in geriatric care, a reliable verbal communication is crucial to the outcome of a treatment, targeting the restoration of self-sufficient life. Studies have shown the negative effect of an unrecognized and unadjusted hearing impairment on the outcome of the geriatric assessment (Barthel-Index, GDS, TuG, Tinnetti, MMSE etc.) that can also lead to a false diagnosis of a dementia due to an unadjusted cognitive testing (MMSE, CERAD, MoCA).

With a reliable, easy to use bedside screening as part of a basic assessment in geriatrics, the hearing impaired patient could be positively screened and the level of awareness among the geriatric staff (nurses, therapist and physicians) could be raised.

Method: Over a six month period 128 of 321 geriatric patients ( 43 male, 85 female, mean age 81 yrs.) that meet the inclusion criteria (inclusion: Geriatric in-patients (age $>70$, consent) exclusion: Geriatric patients (age $<70$ ), unable to use the iPad due to poor vision, MMSE $<12$, consent withheld) were screened with the three-digit screening test, using an iPad IV and Sennheiser HAD 280 earphones, in a bedside situation on the ward. Each patient was afterward included in the geriatric hear care service (GHCS) a subjected to an ENT exam and a pure-tone audiometry for reference. Apart from that, the patient and the nurse/therapist filled in a questionnaire to judge the test concerning applicability, handling and if they thought that the test would be helpful for them.

Results: Nearly $40 \%$ of the participants found the threedigit-test straight forward, but only one third was able to use it without external help. $73 \%$ found the test result 
congruent to expectations concerning their hearing ability. Unfortunately, more than $45 \%$ of the participants were unlikely following up on a pathological result.

From the view of the nurses/therapist $63 \%$ needed help to use the test.

97\% of all participants showed a "pathological test result" (red light), which complies to a high extent with the puretone audiometry results. But as a screening test, its ability to distinguish between a light, medium or severe hearing impairment seems limited.

Conclusions: The three-digit online test is a feasible, easy to use bedside test for a geriatric setting. Nevertheless the test procedure is unlikely to be undertaken by the patient unassisted. Apart from that the likelihood of a follow up with an ENT physician or an audiologist, regarding a hearing impairment and its treatment, was not enhanced by a pathological test result.

\section{The Importance of Social Connections in Rehabilitation for Older Adults}

\section{Sperandio}

MED-EL GmbH, Innsbruck, Austria

This presentation looks at current knowledge about the results of rehabilitation in cochlear implantation for older adults, and then to suggest ways this knowledge can inform the rehabilitation practice. Emphasis is on the importance of social connections in this process, both in the achievements of older adults and in their particular needs in rehabilitation.

It is well known that for older adults social connections diminish with both age and hearing loss. One of the key objectives in cochlear implantation is the restoration of opportunity for communication with others. This is, therefore, an important consideration in the rehabilitation process for this population, and one which is often overlooked.

The emphasis in this presentation is on practical suggestions for clinicians as they consider best practice in rehabilitation for this population. Participants will gain suggestions for their practice which they will be immediately able to implement on return to their practice.

\section{Age-Related Changes to the Auditory Periphery}

\section{J. Dubno}

Medical University of South Carolina, Dept. ORL, Head and Neck Surgery, Charleston, USA

A significant result from studies of age-related hearing loss involves the degeneration of the cochlear lateral wall, which is responsible for producing and maintaining the endocochlear potential (EP). Age-related declines in the EP systematically reduce the voltage available to the cochlear amplifier, which reduces its gain more so at higher than lower frequencies. This "metabolic presbyacusis" largely accounts for age-related threshold elevations observed in laboratory animals raised in quiet and may underlie the characteristic audiograms of older humans: a mild, flat hearing loss at lower frequencies coupled with a gradually sloping hearing loss at higher frequencies. These naturally occurring age-related changes to the auditory periphery in older adults combine with damaging effects of a lifetime of environmental exposures and disease processes. Subsequent anatomic and physiologic deficits result in reduced detection for low-level signals (hearing loss) and impaired suprathreshold auditory function, including complex signal processing and speech understanding. As such, the aging auditory periphery delivers degraded signal representations for processing by the central auditory pathways and cortex, which may impose increased cognitive demands from an aging brain with already limited resources and loss of inhibition. These complex and interactive effects throughout the aging auditory system highlight an important role for auditory plasticity and the critical need for evidence to identify promising targets for intervention and strategies to prevent or delay the onset of age-related declines. (Work supported by NIH/NIDCD).

\section{The Role of Inflammation and Microglial Priming in Age-Related Hearing Loss}

\section{Verschuur}

University of Southampton, Institute of Sound \& Vibration Research, Southampton, UK

Chronic inflammation associated with the ageing process, known as inflammaging, is known to be a contributor to a number of age-related conditions and is implicated as a key mechanism of ageing itself. At the same time, inflammation is known to cause or contribute to other forms of hearing loss including loss of hearing after cochlear implantation. We previously investigated the hypothesis that inflammaging may be a contributor to age-related hearing loss by examining data from the Hertfordshire Ageing Study, a large population cohort of individuals born in the UK in the 1920s [Verschuur et al., 2012]. Analysis showed that there was a significant association between inflammatory markers and hearing level among 611 participants aged 63 to 73 , indicating a link between levels of chronic inflammation and worse hearing outcomes. The association was independent of other known contributors to hearing level such as age, gender, smoking and noise exposure. In order to establish if this finding could be replicated in a separate, unrelated, population cohort and also to extend the investigation to an older age group, we analysed data from the Medical Research Council National Study of Hearing, a large population study of hearing status in the UK. This showed a significant association between (worse) hearing level and (higher) white blood cell count in 320 participants aged 60 to 89 ; the association was markedly greater among those 75 and older.

Having corroborated the association (between age-related hearing loss and inflammatory status), our further aim was to investigate the likely role of neuro-inflammation a plausible mechanism underlying the association. Neuro-inflammation refers to the change in structure and function 
of the microglia, which are the main immune cells of the central nervous system. These changes "prime" the microglial cells which are then ready to mount a more robust immune response given other immune challenges. Systemic inflammation is known to drive or accelerate neurodegenerative changes (as in Alzheimer's disease) via priming of microglia but very little previous work has characterised microglial populations and their role in the auditory system. We hypothesised that age-related hearing loss may be associated with microglial priming in both the peripheral and central auditory system and that this mechanism might underpin any association between chronic inflammation and hearing loss. We will report on our recent histopathological findings from C57BL/6J mice comparing microglial numbers and priming in the cochlear nucleus and cochlea of younger and older mice (who develop an accelerated form of age-related hearing loss). Results will be discussed in the context of a proposed new biological mechanism underlying age-related hearing loss.

\section{Genetics of Age-Related Hearing Loss}

\section{S. Usami}

Shin-ichi Usami, Shinshu University School of Medicine, Matsumoto, Japan

Presbycusis (age-related hearing impairment: ARHI) is the most common sensorineural impairment in elderly people and in developed countries, more than $30 \%$ of people over 65 years old are affected.

It has been thought that any type of hearing loss is caused by (either or both) genetic factors and environmental factors, though influenced ratio of genetic/environmental is variable. For example, congenital hereditary hearing impairment is nearly $100 \%$ genetically determined disease, though injury or viral infection is caused by predominantly environmental factors.

ARHI is situated between, and believed to be a typical complex disorder associated with both genetic factors and environmental factors ("complex ARHI"). The accumulated external and internal factors lead to degeneration and age-related changes in the cochlea. These environmental factors, including exposure to noise for a long time, ear disease, ototoxic drugs, circulatory disease, and diabetes mellitus, play important causative roles in presbycusis. The effect on the development of presbycusis of smoking and alcohol is controversial.

On the other hand, some hereditary hearing loss patients showed late-onset hearing loss similar to presbycusis ("monogenic ARHI"). Recently we reported a small portion of patients with $\mathrm{CDH} 23$ mutations exhibiting ARHI-like phenotype [Miyagawa et al., 2012, Usami et al., 2012]. In addition to $\mathrm{CDH} 23$, our recent genetic analysis identified some responsible genes such as $\mathrm{COCH}$ also showed ARHI-like phenotype. These data strongly supports the view that there are at least two types of ARHI and a particular type of ARHI (late onset hereditary hearing loss) is monogenically inherited.

\section{Tuning the Brain for Communication}

\section{N. Kraus}

Northwestern University, Department of Neurobiology, Evanston, USA

The functioning of the entire auditory system, comprising the classic auditory pathway as well as the cortical, cognitive centers that influence it, underlies successful communication. With hearing loss, communication suffers. Fortunately, the auditory system is dynamic, with its dynamicity occurring over three broadly-defined timescales: the course of a lifetime; shorter periods-weeks, months or years of training; and even online in-the-moment adaptation to characteristics of sounds in one's environment.

I will be speaking today about training-induced changes on the middle time scale, in particular, focusing on studies from my group that have looked at the effects of musical training and computer-based training activities on older adults with and without hearing loss. The neural metric we employ is the complex-stimulus-evoked auditory brainstem response (cABR). Its characteristics, such as timing, magnitude, consistency, phase, pitch tracking, envelope tracking, and more, are affected by cognitive influences on the auditory system. As such, it is a valuable lens through which to explore auditory system tuning brought about by various types of training. Concurrently, we measure facets of communication such as memory, attention and hearing in noise. Improvements in these areas are examined in light of the neural changes that accompany them.

We also have examined the effect that hearing loss has on the pattern of change. Both modes of training are relevant to both technological and artistic solutions for strengthening auditory and cognitive skills when hearing loss is present. From a basic science standpoint, results bolster our understanding of the integration of cognitive centers with that of the classical auditory system. They also provide a platform and a method for investigation of the neural plasticity that can be accomplished with training via multiple routes and timeframes.

\section{Questions for "Auditory Training: Approaches and Outcomes"}

\section{Moore}

Cincinnati Children's Hospital, Ohio, USA

Abstract: Cognition makes a major contribution to hearing, especially for complex signals (e.g. speech) in challenging environments. Hearing loss of peripheral origin is currently addressed through amplification or electrical stimulation of remaining intact elements. These techniques provide sensory enhancement, and there is evidence that they can also impact positively on cognition. Another mechanism for improving the processing of sensory signals is through training. Training occurs 'naturally' when hearing aid or cochlear implant users interact with their auditory world following 'turn on' of their new devices. Evidence from laboratory studies of perceptual learning 
suggests that this training primarily or exclusively works through improved cognitive processing. For example, we have shown that auditory discrimination and updating working memory training provide reciprocal benefit that appears to be governed by supramodal processes. However, working memory training was more broadly transferrable to auditory learning. This suggests that identifying and training key cognitive skills may be a more effective strategy than training simple auditory discrimination, a technique that has dominated largely unsuccessful efforts in auditory training to date. Examples of successful training strategies in vision, for treating amplyopia and visuomotor skills, point the way to more successful auditory learning, both with and without the use of hearing devices.

\section{Cortical Resource Allocation in Older Adults} with Hearing Loss

\section{A. Sharma}

University of Colorado at Boulder, USA

A basic tenet of neuroplasticity is that the brain will re-organize following sensory deprivation. Sensory deprivation appears to tax the brain by changing its normal resource allocation. A better understanding of the cortical re-organization that accompanies hearing loss may allow us to incorporate improvements in the design of prostheses to allow them to better accommodate altered cortical processing. Compensation for the deleterious effects of hearing loss may include recruitment of alternative or additional brain networks to perform auditory tasks. Our highdensity EEG experiments suggest that adult-onset hearing loss results in significant changes in neural resource allocation, reflecting patterns of increased listening effort and decreased cognitive reserve, which may be associated with dementia-related cognitive decline. Cross-modal plasticity is another form of cortical re-organization associated with deafness. Cross-modal plasticity occurs when an intact sensory modality recruits cortical resources from a deprived sensory modality to increase its processing capabilities as compensation for the effects of sensory deprivation. Deaf animals show recruitment of higher-order auditory cortical areas, by visual and somatosensory modalities resulting in enhanced capabilities for the recruiting modality, with likely negative consequences for auditory perception. Our results in humans suggest clear evidence of recruitment of higher-order auditory cortical areas by visual and somatosensory modalities in both congenital deafness and adult-onset hearing loss. Cross-modal cortical re-organization is evident in early stages of hearing loss and shows a strong negative correlation with speech perception outcomes, suggesting that recruitment by visual and/or somatosensory modalities may influence the variability in outcomes seen in implanted patients. Overall, our results suggest that compensatory cortical plasticity secondary to sensory deprivation has important neurological consequences and influences outcomes for hearingimpaired individuals.

\section{Temporal Processing in Elderly CI Listeners}

\section{Friesen}

Toronto, Canada

Because more individuals with hearing loss are being treated with a cochlear implant (CI), it is important to understand how aging affects physiological mechanisms underlying CI listening. It has been clinically observed that when older CI users' performance is poor, they tend to prefer listening with slower CI stimulation pulse rates. We hypothesized that this preference may reflect agingrelated loss of neural synchrony with higher rate stimulation, which may in turn affect speech understanding. Auditory evoked potentials reflect neural activity related to stimulus processing in the auditory cortex. In particular, the N1-P2 complex (responses at 50-200 ms after stimulus onset) is known to be sensitive to age-related changes in acoustic feature processing such as the temporal properties of the stimulus. Therefore, the aim of this study was to determine whether the physiological representation of stimulation pulse rate as indexed by the N1-P2 complex changes with age in CI users.

Seven elderly CI listeners (aged 70 to 85 years) and 7 younger CI users (aged 40 to 55 years) having the Advanced Bionics HiRes 90k device were tested. Auditory evoked potentials were recorded from the subjects while they received electrical stimulation consisting of a repeated pulse train of $200 \mathrm{~ms}$-second duration presented directly to a median electrode of the CI device. The pulse rate was varied using $3000,2000,1000$, and 500 pulses per second (pps) in separate blocks. Amplitudes and latencies of the N1 and P2 waves were examined for the different pulse rates and between the two age groups. Results will be discussed.

\section{Auditory-Cognitive Processing in Older- Adult Cochlear Implant Users}

\section{Y. Henkin, Y. Yaar-Soffer, M. Steinberg, C. Muchnik}

Sheba Medical Center, Tel Hashomer, Tel Aviv University, Israel

A growing body of literature suggests that older adults benefit from cochlear implants (CI). Nonetheless, variability in hearing performance is high, and conflicting evidence exists as to whether older CI recipients have similar outcomes as compared to young CI recipients. Evaluation of CI benefit is predominantly performed by means of speech perception tests that are sensitive, to some degree, to the difficulties encountered by older CI recipients. They do not reflect, however, cognitive aspects of speech understanding that are known to deteriorate with age, and affect the amount of attention, effort, and memory resources expended during communication. A potential objective means for assessing the benefits derived from CIs in older adults are electrophysiological measures. In particular, auditory event-related potentials (AERPs) that allow evaluation of the time-course of cortical information processing, from early perceptual to later cognitive, post-perceptual stages may prove advantageous. By utilizing complex tasks with enhanced cognitive load during 
AERPs recordings, processing difficulties of older CI recipients may be exposed. The goal of the present study was, therefore, to assess auditory-cognitive processing in older-adult CI recipients by means of AERPs and behavioral measures during Stroop tasks. AERPs were recorded from 32 scalp electrodes from post-lingually deafened older- and young adults with CI and from normal hearing $(\mathrm{NH})$ age-matched listeners. Participants performed Stroop tasks requiring classification of word meaning or speaker's gender while ignoring the irrelevant (congruent or incongruent) speaker's gender or word meaning, respectively. Attention was assessed in all participants by means of the digit span test (forward and backward), and older adults (CI and $\mathrm{NH}$ ) filled the mini-mental state examination questionnaire. While data collection is still in progress results indicate that auditory-cognitive processing in older $v s$. young $\mathrm{NH}$ adults was characterized by: 1 ) Similar Stroop effect magnitude and performance accuracy; 2) Preserved sensory - perceptual processing (P1, N1, P2); 3) Prolonged higher-order perceptual processing (P3), response selection, and execution time; and 4) Absent neural events reflecting higher-order perceptual (N2) and post-perceptual (N4) processing. Comparison between auditory - cognitive processing of older CI users and older $\mathrm{NH}$ listeners revealed similar findings with respect to Stroop effect magnitude, sensory-perceptual processing (N1), and absence of perceptual (N2) and postperceptual (N4) neural events. In contrast, performance accuracy was poorer the timing of higher-order perceptual processing (P3 latency) was substantially prolonged in CI users. Taken together, AERPs and behavioral measurements during Stroop tasks unraveled the effects of age, hearing loss, and limitations of the CI device on auditory - cognitive processing.

\section{Cochlear Implant Surgery and Revisions}

\section{H.C. Pillsbury, M.T. Dillon}

University of North Carolina Hospitals and Clinics, Chapel Hill, USA

Candidacy for cochlear implantation is dependent on unaided residual hearing thresholds, speech perception outcomes with appropriately fit amplification, and health to undergo surgery. Rarely is age considered a contraindication to cochlear implantation candidacy. In fact, many implant recipients are implanted after 65 years of age. Although special consideration regarding the patient's health is considered, these patients find continued success with cochlear implantation over their previous listening condition.

Surgical considerations for the older adult population versus younger adults at the initial surgery and in cases of revision cochlear implantation will be discussed. Additional considerations include results of preoperative blood work and cognitive function, and postoperative pain control and urinary function.

Further, the speech perception outcomes of adults who underwent revision cochlear implantation will be reviewed. Speech perception abilities as measured with CNC words were retrospectively reviewed. Older adults who underwent revision cochlear implantation after 65 years of age experienced a similar restoration in speech perception abilities 3 and 6 months post-revision as a younger adult cohort.

There are similar surgical considerations for older adults at the initial cochlear implantation and in revision cases. When revision surgery is warranted, older adults may experience a similar restoration in speech perception abilities as compared to younger adults. Advanced age in cases of hard or soft failures should not be considered a contraindication to revision surgery.

\section{Hearing Preservation in Older Adults with Cochlear Implants (EAS) and Middle Ear Implants}

\section{A. Wolf-Magele ${ }^{1,2}$, J. Schnabl ${ }^{1}$, G.M. Sprinzl ${ }^{1,2}$}

\section{${ }^{1}$ Landesklinikum St.Pölten, Department of Otorhinolaryngology, Head and Neck Surgery, Austria \\ ${ }^{2}$ Karl Landsteiner Private University, Austria}

Introduction: Today the preservation of residual hearing of the low frequencies is a standard approach for cochlear implant centres and is part of the clinical routine meanwhile. Hearing preservation is not only important in electric acoustic stimulation (EAS), it is especially in an active middle ear implantation (AMEI) a requirement in order to take advantage later on. Nowadays there are many people, worldwide about 278 million people, who suffer from moderate to profound hearing impairment in both ears. The prevalence increases with age, and more than one third of people older than 65 years have a significant hearing loss. In our presentation we show the outcome of hearing preservation (in EAS and AMEI) especially in elderly patients (more than 60 years).

Method: In the presentation the current knowledge of hearing preservation is summarized. Different techniques are presented for hearing preservation. The benefits of EAS are discussed and different electrodes types for hearing preservation are presented. Minimal invasive approaches to the inner ear as well as the use of atraumatic electrodes are the preconditions to achieve the goal of successful hearing preservation in adults and children. The additional administration of corticosteroids is helpful and may influence the success rate of hearing preservation. Audiological test were done perioperative and postoperative with a follow up to 5 years. The age distribution our implant users are represented separated in age groups (60-70, $71-80,+81$ years).

Results: Since 2008 overall 344 patients received a hearing implant in Innsbruck and St. Pölten. 129 patients were implanted with an AMEI. 60 (46.5\%) were older than 60 years; 29 (22.5\%) of them were older than 70 years. 10 patients of our 215 CI cases got an EAS; 4 of them were older than 60 years. All of our patients were satisfied with their implant. No peri- or postoperative complications occurred. In two cases residual hearing could not be obtained after 1 year of using. Compared to the younger patients, no differences can be shown. 
Discussion: Preservation of residual hearing is also in the elderly population a reliable option in cochlear and active middle ear implantation. To choose the right technique is equally important as the use of the atraumatic electrodes. Age is no argument against the hearing preservation technique, however in the elderly special protocols are required to achieve satisfying hearing preservation rates. It is an important issue to check that the hearing loss is stable since duration of minimum 2 years. To create the best conditions for the patients and the surgery before can influence the success rate of hearing preservation in a promising way.

\section{Hearing Preservation in Older Adults with Cochlear Implants}

\section{K.M.J. Green, D. Mawman, I.A. Bruce}

University of Manchester, UK

Introduction: Hearing preservation cochlear implant surgery is well established and the benefits of this are well recognised. We present our results of hearing preservation surgery in older adults using a cochleostomy approach and deep insertion using MED-EL Flex electrodes (28 $\mathrm{mm}$ and $31 \mathrm{~mm}$ ).

Methods: A retrospective study was undertaken of older adults undergoing CI with hearing preservation at our institution. 10 patients were included in the study, and the main outcome measure was pure tone thresholds after surgery.

Results: The study group consisted of 10 adults with an average age at implantation of 72.1 years (range 68 to 76 years). All of the patients had electrode insertion via the cochleostomy approach. 4 patients had a Flex Soft electrode $(31 \mathrm{~mm})$ and 6 had a Flex 28 electrode $(28 \mathrm{~mm})$. One patient had a complete loss of residual hearing after surgery and all of the remaining patients had preservation of hearing. The average deterioration at $125 \mathrm{~Hz}$ was 17.8 $\mathrm{dB}$, at $250 \mathrm{~Hz}$ was $18.9 \mathrm{~dB}$, at $500 \mathrm{~Hz}$ was $12.2 \mathrm{~dB}$, and at $1000 \mathrm{~Hz}$ was $4.2 \mathrm{~dB}$. None of the patients had a deterioration in hearing of $>40 \mathrm{dBHL}$

Conclusions: This study demonstrates that residual hearing can be preserved in older adults using a cochleostomy approach and deep insertion of Flex electrodes. The results are comparable to those seen in younger adults and adolescents.

\section{An Electric Complement to Treat Certain Types of Presbycusis}

\author{
P.H. Skarzynski ${ }^{1,2,3}$, A. Lorens $^{1}$, H. Skarzynski ${ }^{1}$ \\ ${ }^{1}$ World Hearing Center, Institute of Physiology and Pathology of \\ Hearing, Warsaw/Kajetany, Poland \\ ${ }^{2}$ Ophthalmic Diagnostics and Rehabilitation and Sensory \\ Organs Department, Medical University of Warsaw, Warsaw, \\ Poland \\ ${ }^{3}$ Institute of Sensory Organs, Kajetany, Poland
}

There is a large population of elderly with significant hearing impairment for whom auditory rehabilitation with state-of-the-art hearing aids is inadequate. The auditory deprivation in these patients leads to social isolation and significant deterioration in quality of life.

The elderly patients of the Institute of Physiology and Pathology of Hearing are a heterogenous group in terms of the etiology of the impairment, as well as pure tone thresholds. Frequently we are confronted with the situations when hearing in low frequency range of elderly patients is normal or fully functional and it is only necessary to complement it electrically in the medium and high frequency ranges. The first CI surgery during which an existing normal hearing had been electrically complemented was performed in 2002 in the Institute and new method of Partial Deafness Treatment (PDT) was established. The standard surgical procedure in PDT is the round window hearing preservation approach [Skarzynski et al., 2003, 2006, 2007, 2009, 2010, 2012].

The paper will comprise a retrospective analysis of hearing preservation and speech audiometry results after cochlear implantation in elderly patients over 60 years of age when the low frequency hearing was complemented by electrical stimulation (Electric Complement - EC).

\section{Is There an Age Limit for Cochlear Implantation?}

\section{Lassaletta}

Hospital La Paz, Madrid, Spain

Objective: To evaluate quality of life following cochlear implantation in elderly postlingually deaf adults.

Methods: Postlingually deaf adults 60 year-old or older who underwent cochlear implantation in 3 institutions. Data concerning demographics and audiometric evaluation were studied. The Glasgow Benefit Inventory was used to quantify the quality of life. Patients were divided in two groups ( $<70$ and $\geq 70$ years) and results were also compared to younger adults recipients ( $<60$ years).

Results: Eighty-one patients were included in this study. The mean age at implantation was 68 years (range 60-82). Cochlear implantation in the elderly improved audiometric outcomes (pure tone average and speech perception) significantly $(p<0.01)$. The results of the GBI showed an Overall Benefit of cochlear implantation in elderly $(+36)$ and on the individual subscales $(+49 ;+20$ and +1$)$. Quality of life difference between the $<70$ and $\geq 70$ year groups was not significant $(\mathrm{p}=0.90)$. These results were similar to those of younger postlingually implanted recipients.

Conclusions: Elderly cochlear implant users improve their quality of life, with similar outcomes to those achieved in younger adults. Due to the prevalence of age-related conditions in the elderly, which may increase the risk for surgery, particular attention must be paid when indicating a cochlear implant. 


\section{Image-Guided Programming for Improving Speech Understanding and Spectral Resolution in Younger and Older Implant Recipients}

\author{
R. F. Labadie, J. H. Noble, R. H. Gifford, A. Hedley- \\ Williams, B. M. Dawant
}

Vanderbilt, Bill Wilkerson Center, Nashville, USA

Cochlear implants (CIs) are arguably the most successful neural prosthesis to date. Despite this success, a significant number of CI recipients experience marginal hearing restoration, and, even among the best performers, restoration to normal fidelity is rare. We have developed CT image processing techniques that can be used to detect the positions of implanted CI electrodes and quantify the electrode-to-neural interface for individual CI users. These techniques permit development of new, patient-customized CI processor programming strategies. In this presentation, we will present one such strategy that uses this patient-specific spatial information to decrease cross-electrode channel interactions, and we will show that this new strategy improves hearing outcomes in a study conducted with $60 \mathrm{CI}$ recipients (23 bilateral, 37 unilateral) for a total of $64 \mathrm{CI}$ reprogrammed.

Prior to enrollment, each of the experienced CI users (length of CI use 0.4-14.7 yrs, average $=2.9 \mathrm{yrs}$ ) had reached asymptotic hearing performance via traditional behavioral programming approaches. Hearing performance was quantified for CNC words and phonemes, AzBio sentences in quiet and noise $(+10 \mathrm{~dB}$ SNR) and BKB-SIN sentence recognition in noise. In addition to the standard measures of speech recognition, we assessed spectral modulation detection (SMD) at 0.5 and $1.0 \mathrm{cy}$ cle/oct using a method of constant stimuli. For each subject, hearing performance was assessed with his/her existing clinical program prior to remapping. Subjects used the experimental map based on our individualized position-based model for a period of 3-4 weeks after which hearing performance was again assessed.

Average performance demonstrated statistically significant improvement on all measures with wide variation in individual responses. Perhaps the most noteworthy metric of improvement is that 55 of $64(86 \%)$ elected to retain the experimental map.

We believe these results are clinically significant because: (a) this new strategy is compatible with the majority of existing cochlear implant systems; (b) it drastically improves hearing outcomes independent of device type; and (c) we believe this opens the door to a new avenue of research into other more comprehensive image-guided programming strategies that could further improve hearing outcomes in CI recipients.

This work was supported by grants R21DC012620, R01DC008408, and R01DC009404 from the NIDCD and UL1TR000011 from the National Center for Advancing Translational Sciences.

\section{Combining Acoustic, Electric and Visual Information by Older Adults with Cochlear Implants}

\author{
M. Dorman 1 , S.J. Cook ${ }^{1}$, R. Gifford ${ }^{2}$ \\ ${ }^{1}$ Arizona State University, Arizona, USA \\ ${ }^{2}$ Vanderbilt University, Nashville, USA
}

Recently we described the ability of cochlear implant (CI) patients, ranging in age from 19 to 81 years, to combine two sources of information about speech when those sources were (i) two cochlear implants or (ii) a cochlear implant and low-frequency acoustic hearing from the contralateral ear [Dorman et al., J. Hear Sci, 2(4), 2012]. Plots of benefit when adding the 'second' source of information to the first, suggested that the physiological processes correlated with ageing did not alter the benefit obtained from adding a second implant or low-frequency information from the contralateral ear. In a new series of experiments we have increased the information available to CI patients by including information from vision in the stimulus set. Our initial results suggest that the presence of visual information about speech significantly alters the value of both a 'second' CI (for bilateral patients) and alters the value of low-frequency acoustic hearing for patients with a CI and either ipsilateral or contralateral acoustic hearing. For most patients, information from vision potentiates the value of the second CI but decreases the value of low-frequency acoustic information.

\section{Long-Term Speech Perception in Elderly Cochlear Implant Users}

\section{Dillon, E. King, M. Adunka, H. Pillsbury, C. Buchman, O. Adunka}

University of North Carolina at Chapel Hill, North Carolina, USA

Advanced age alone is rarely considered a contraindication for conventional cochlear implantation. Understanding the acclimatization of speech perception specific to patients implanted after 65 years of age may improve realistic expectations during preoperative counseling. Patients with preserved low-to-mid frequency residual hearing postoperatively may experience different outcomes by listening to the combination of acoustic and electric stimulation.

Two cochlear implant recipient groups were evaluated: conventional cochlear implant recipients without hearing preservation and clinical trial participants with hearing preservation. The conventional cochlear implant recipients were all implanted after 65 years of age and had long-term ( $\geq 10$ years) listening experience with the external speech processor. Speech perception performance as measured with CNC words in quiet and HINT sentences in quiet and steady-state noise were reviewed over time. The second group participated in the Electric-Acoustic Stimulation (EAS) clinical trial and was implanted with the FlexEAS electrode array. Subjects with preserved residual hearing in the implanted ear were fit with acoustic and electric stimulation in an ipsilateral listening condition. Speech perception abilities were assessed with CNC words 
in quiet and CUNY sentences in steady-state noise, and compared between younger and older adult ( $\geq 65$ years) EAS recipients.

On average, patients implanted after 65 years of age do not experience a decline in speech perception performance with extended listening experience and may potentially continue to see improvement beyond the 12-month follow-up interval. Younger adult EAS recipients initially show a trend of having better speech perception performance than an older adult cohort. Long-term outcomes in the older adult EAS population are needed as candidacy criteria broaden to include patients with greater amounts of low-to-mid frequency residual hearing.

\section{Music Perception in Older Adult Cochlear Implant Users: Insights from a European Multicenter Study}

\section{Georgios Papadelis}

Department of Music Studies, School of Fine Arts, Aristotle University of Thessaloniki, Greece

Studies in the last two decades or so that looked at music habits of Cochlear Implant (CI) users and their enjoyment of music has shown a gradual increament of the amount of time CI users spend listening to music postimplantation on an everyday basis, and higher levels of satisfaction with the musical sound they experience with their implants as compared to users of older generation CI systems. Although these results are encouraging, performance of CI recipients on music listening tests suggest that even with current-day implant technologies, accuracy in perceiving fundamental attributes of the musical sound, such as pitch and timbre, still remains suboptimal. However, poor pitch sensitivity does not appear to severely affect perception of melodies, since processing of melodies and familiar tunes frequently relies on gross features of a melody, such as the melodic contour, or even on characteristic aspects of their rhythmic structure. Processing of more complex structures in music such as chords or harmonic sequences still remains a challenging issue for implant designers. In contrast, CI recipients demonstrate close-to-normal temporal sensitivity, and similar accuracy as normal hearing adults in perceiving the rhythmic aspects of music.

While music perception in adult CI users has been extensively investigated up to the present time, there is only limited research on the effects of age-related deterioration of auditory processing and general cognitive functions on music listening abilities of elderly CI users. In this talk, empirical evidence on potential associations between CI users' chronological age and their performance on music listening tests is presented, together with data on the influence of aging on the role that music plays in their lives postimplantation. Analysis is based on data from a recently completed European multicenter study on music perception in CI users wearing a PULSAR ${ }_{\mathrm{CI}}^{100}$ or a SONATA ${ }^{100}$ with an OPUS 2 audio processor $(\mathrm{N}=81$, age range: $21.2-82.3$ years), and Normally Hearing $(\mathrm{NH})$ adults ( $\mathrm{N}=46$, age range: $21.1-58.8$ years). Areas of music listening abilities that display noticeable declines in older implant users are highlighted by comparing distributions of test scores among a subgroup of CI users over 60 years $(\mathrm{N}=24)$, the subgroup of users younger than 60 , and the group of $\mathrm{NH}$ controls.

Results showed that older adult CI users were equally accurate to younger ones in identifying the direction of pitch-changes of musical tones, in identifying musical instruments that play either in solo or in ensembles, and in detecting differences between melodies or chords. In contrast, older adult CI users appear to be significantly worse in discriminating between different rhythm patterns, in recognising monosyllables, and gave significantly lower ratings of the role of music in their lives. In summary, these results indicate that general slowing of temporal processing with aging, which mainly associates with more central timing mechanisms of the auditory nervous system, may constitute a common underlying factor contributing to the obtained rhythm and speech perception decline in elderly CI users, while more peripheral deficiencies in frequency analysis and sensitivity, attributed to the functionality of the implant system in conjunction with the impaired inner ear, do not appear to vary considerably over time.

\section{Outcomes in Geriatric Cochlear Implantation}

\section{Friedland, C. Runge}

\section{Medical College of Wisconsin, Milwaukee, USA}

As the geriatric population expands, and individual longevity increases, cochlear implant candidates are being implanted at older ages, often into their ninth and tenth decades. While cochlear implantation is an effective intervention for older individuals with advanced presbycusis, it is important to understand expected outcomes and limitations of performance that may come with older age. Performance of 78 patients older than 65 was evaluated for an effect of age on 1-year post-implantation word and sentence scores. There was no age-related difference regarding pre-operative duration of deafness or audiometric measures. Post-operatively age had no significant effect on CNC or HINT/Q performance but a significant age-related correlation was noted for HINT/N scores $(\mathrm{p}<0.05, \mathrm{r}=0.40)$. The strongest predictor of post-operative performance on all tests was pre-operative scores on HINT/Q regardless of age. In order to further assess geriatric cochlear implant performance, 1-year post-implantation scores on sentence and word tests of patients older than 65 years was compared to an audiometrically matched cohort of younger adults ( $\mathrm{n}=28$ in each cohort). Both groups showed substantial improvement in speech recognition in both quiet and noise. Older individuals, however, performed slightly worse on CNC and HINT/Q tasks than younger subjects at 1-year post-implant. Within the older cohort HINT/N performance trended toward poorer performance and there was wider variability of performance in noise within the older cohort than the younger group. These data demonstrate the efficacy of cochlear implantation in improving speech perception in older candidates. However, they suggest that expected outcomes for older individuals should be tempered as compared to those expected in audiometrically similar younger adults. We will discuss these 
data and their implications related to the timing of intervention for older adults, candidacy criteria for the older patient, and considerations regarding pre-implant assessment of central auditory processing and cognitive function.

\section{Epidemiology of Vestibular Dysfunction}

\section{Y. Agrawal}

Johns Hopkins University School of Medicine, Baltimore, USA

The vestibular system is integral to balance control and locomotion. In this session we will review the different components of the vestibular system, discuss ways to measure of vestibular function, and evaluate studies of the magnitude and distribution of vestibular dysfunction in the population. We will focus particularly on age-related vestibular loss, and implications for mobility and falls risk in older individuals.

\section{Assessment and Treatment Options in Age-Related Vertigo and the Prevention of Falls}

\section{A. Ernst}

\section{Dept. Otolaryngology at UKB, Berlin, Germany}

Age-related vertigo (presbyvertigo) is characterized by a functional and structural decline in the vestibular, the optic and the musculoskeletal systems, respectively [Baltes and Mayer 2001]. It leads to gait and stance instability which finally can induce falls and is related to a decline in hearing as well [Zuniga et al. 2012].

There is evidence in the literature that a careful dietary, drug intake and exercise regime can improve balance [Roberts et al. 2013], but in general the programs in preventing falls utilizing those and other common-sense measures (e.g., abolishing slippery ground at home) are not very effective (only $9 \%$ reduction in falls) [Choi et al. 2012].

Usually, balance maintenance in the elderly is clinically assessed by screening tests (e.g., Tinetti's test). There are other clinically proven tests (analysis of body sway) available which use a standardized test battery (gSBDT) to analyse the risk-to-fall [Basta et al. 2012]. Since conventional vestibular rehab requires a long-term, often exhausting training and has a low rate of success [Howe et al. 2011], an individualized neurofeedback (NFT) training which is based on an analysis of body sway in everyday-life conditions seems significantly more effective [Basta et al. 2011]. The present study was aimed at investigating this approach in a double-blind placebo-controlled study design. Fifty-four patients with presbyvertigo performed the vibrotactile NFT ( 2 weeks, $15 \mathrm{~min} /$ day) based on an individual body sway analysis in 14 everyday-life stance and gait conditions (20 s each). The basic principle of NFT is to give the patient an intuitive, vibrotactile signal at the hip in that direction which showed a higher body sway than the preset threshold. The mean reduction of sway was $36.0 \%$ in pitch and $34.0 \%$ in roll directions, respectively. Significant improvements were also observed in platform stability measurements (composite score of the Sensory Organization Test) and in questionnaires (DHI/VSS). No significant changes were found in the placebo group (similar training with random feedback signal).

The present data shows that NFT with the small, bodyworn Vertiguard ${ }^{\oplus}$-RT device improves the balance in everyday-life conditions significantly with minimal efforts for the patients. This new method seems to be well-suited for home or group training in the elderly to prevent falls and improve overall postural stability.

\section{Evaluation of the relative benefits of cochlear implantation according to the level of residual hearing}

\section{A. Lorens ${ }^{1}$, B. Wilson ${ }^{2}$, A. Piotrowska ${ }^{1}$, H. Skarzynski $i^{1}$, P.H. Skarzynski $i^{1,3,4}$}

\author{
${ }^{1}$ World Hearing Center, Institute of Physiology and Pathology of \\ Hearing, Warsaw/Kajetany, Poland \\ ${ }^{2}$ Duke University and Duke University Medical Center, \\ Durham, NC, USA \\ ${ }^{3}$ Ophthalmic Diagnostics and Rehabilitation and Sensory \\ Organs Department, Medical University of Warsaw, Warsaw, \\ Poland \\ ${ }^{4}$ Institute of Sensory Organs, Kajetany, Poland
}

A retrospective chart study was conducted to evaluate the benefits of cochlear implantation according to levels of residual hearing at low frequencies. The charts for $159 \mathrm{pa}-$ tients were culled from the archive of charts at the ICHS. The criteria for selection were: (1) measurable residual hearing and (2) use of that hearing in conjunction with a cochlear implant following the operation. The residual hearing that was used after the operation was categorized as good $(-55 \mathrm{~dB} \mathrm{HL}$ or better at $500 \mathrm{~Hz}$, or a PTA of $45 \mathrm{~dB}$ HL or better for 125,250 , and $500 \mathrm{~Hz})$, moderate $(-80 \mathrm{~dB}$ $\mathrm{HL}$ or better at $500 \mathrm{~Hz}$, or a PTA of $70 \mathrm{~dB} \mathrm{HL}$ or better), or poor (worse than $80 \mathrm{~dB} \mathrm{HL}$ at $500 \mathrm{~Hz}$ and PTA worse than $70 \mathrm{~dB} \mathrm{HL}$ ). The charts included demographic, audiometric, and speech reception data. The speech reception tests included recognition of monosyllabic words presented in quiet and in competition with noise at the speech-tonoise ratio of $+10 \mathrm{~dB}$. The listening conditions included: (1) use of the residual hearing only, usually with a hearing aid for one or both ears, and (2) use of the residual hearing in conjunction with the cochlear implant.

Among the results was the surprising finding that patients with high levels of residual hearing (the "good" category above) receive benefits from cochlear implantation that are at least as great as the benefits received by the patients with the lower levels of residual hearing. In addition, the highest scores for the speech tests were obtained by the patients with the high levels of residual hearing. These findings are counter to the conventional wisdom that patients with such good residual hearing can be harmed by cochlear implantation and suggest that the criteria for implant candidacy should be relaxed even further so that many more patients can benefit from the procedure.

An additional interesting finding is that the benefits for persons who received their cochlear implants at age 60 or older were at least as great as the benefits for persons who received their cochlear implants earlier in life. Indeed, a 
small but significant reduction in benefits was observed for persons who received their cochlear implants during the teenage years.

These findings together suggest the possibility that sufferers from certain types of presbycusis may benefit from cochlear implantation as well. Age beyond 60 is not a contraindication for cochlear implantation, and only a slight relaxation in the present criteria for implant candicacy could include persons with sensory or sensory + metabolic presbycusis.

\section{Auditory and Cognitive Characteristics of Adults Presenting for Cochlear Implant Workup}

\section{R. Gifford}

Vanderbilt University, Nashville, USA

Managing expectations is a major component of rehabilitative clinical audiology and otology. This is particularly true in the treatment of cochlear implant (CI) candidates preparing to undergo an irreversible surgical procedure. Outcome data showing postoperative speech understanding scores ranging from 0 to 100 percent correct on various measures are of little use in a counseling session. In recent years the issue has been further complicated as more and more aged individuals with measureable hearing in one or both ears are seen for CI evaluations. There have been a number of studies that have shown little to modest correlation between audiometric thresholds, preoperative speech understanding, age, and postoperative speech recognition performance. In cases where a significant correlation was reported, the extreme ends of the function were driving the correlation. The purposes of this study were to 1) characterize the audiologic profile and cognitive status of adults reporting for preoperative cochlear implant workup in a large academic medical center, and 2) to investigate whether the addition of a cognitive screening tool could help guide expectations for postoperative outcomes. 